\title{
A METHOD FOR DETERMINING THE ALKALINITY OF THE BLOOD
}

\author{
By A. E. BOYCOTT, M.A., D.M., Lecturer on Pathology; AND \\ R. A. CHISOLM, M.A., B.M., Greville Research Student. \\ From the Gordon Pathological Laboratory, Guy's Hospital
}

(Received November 29th, 1909)

Apart from the question whether the blood is really alkaline in the sense that it contains such an excess of hydroxyl ions as can be determined by the available physical methods, there is no doubt that the amount of acid which must be added to blood to produce a certain effect with a given indicator varies under different circumstances, and it seems clear that these variations are the expression of phenomena which cannot be neglected. The current methods of determining the alkalinity of blood involve the use of various coloured indicators-litmus, dimethylamidoazobenzol and the like. Since the effect which is produced by the addition of acid to blood is under these circumstances a change of colour, such processes are practically restricted in their application to plasma or serum. This would probably be on the whole rather an advantage for most purposes if the methods were in themselves precise. It seems to us, however, to be very difficult by these means to reach an exact and definite end-point. The ordinary coloured indicators do not give a sharp end-point when used in the titration of proteid solutions. In the first place the actual colour of the indicator may vary materially with the presence or absence of proteid. Serum + congo red will not turn blue after the addition of an enormous excess of strong mineral acid $^{1}$. If two similar quantities of alkali, to one of which is added a little serum or egg-albumin, are titrated with acid in parallel using dimethylamidoazobenzol, it will be seen that at no stage in the process are the tints of the two solutions precisely the same, and it is impossible with any excess of acid to realise in the proteid solution the full red tint of the acid aqueous solution. In the second place, making such allowance as one can for these differences in tint, one finds that the end-point in the presence of proteid ceases to be sharp and is dragged out over a long range of added acid. Thus with dimethylamidoazobenzol

1. Gamble, Journal of Pathology, Vol. XI, p. 134, 1906 ; G. Mann, Physiological Histology, p. $455,1902$. 
10 c.c. $\mathrm{N} / 10 \mathrm{Na}_{2} \mathrm{CO}_{3}+2$ c.c. of 20 per cent. solution of egg white required 11.9 c.c. $\mathrm{N} / 10 \mathrm{H}_{2} \mathrm{SO}_{4}$ to produce the first trace of pinkness and 14.8 c.c. to give a definite red, the end-point extending by indefinite gradations over a range of $2 \cdot 9$ c.c. $\mathrm{N} / 10$ acid or about 20 per cent. of the amount of acid used. Similar experiments with dimethylamidoazobenzol and $\mathrm{NaH} \mathrm{CO}_{3}$ gave a range of $3 \cdot 2$ c.c., with $\mathrm{NaH}_{2} \mathrm{PO}_{4} 2 \cdot 9$ and $2 \cdot 8$ c.c., whereas without the addition of proteid the same titrations had an endpoint range of 0.3 or 0.4 c.c. at most. The indefinite character of the end-point is sufficiently obvious with litmus in using the original method of Wright ${ }^{1}$ and with dimethylamidoazobenzol in the modification introduced by Moore and Wilson ${ }^{2}$. With these methods the difficulty of saying where the colour of the test drop changes is increased by the fact that the periphery of the drop may be one colour and the centre another. The result is that the determination of the end-point is essentially subjective. That this does not necessarily vitiate the methods is of course clearly shown by the concordant data which have been obtained by their use ${ }^{3}$. But it seems to us that with these, as with other methods of a similarly subjective nature, consistent results can only be obtained by a practised observer, and that any isolated observations made when one's 'eye is out' are not altogether free from suspicion of error.

The possibility of dispensing with foreign indicators altogether was suggested by the method of Dare ${ }^{4}$ in which acid is added to diluted laked blood until the absorption bands of oxyhaemoglobin are no longer visible on spectroscopic examination. In its original form this method, as was pointed out by Gamble ${ }^{5}$, gives very vague results and is quite unworkable. 13ut, as we showed some time since ${ }^{6}$, if the temperature at which the reaction takes place is raised, pretty definite end-points are given within a reasonable time by changes in the colour of the haemoglobin and in the gross appearance of the mixtures of dilute blood and acid.

1. Lancet, 1897, Vol. II, p. 719.

2. Bio-Chemical Journal, Vol. I, p. 297, 1906.

3. Moore and Wilson, loc. cit.; W. N. W. Watson, Journal of Pathology, Vol. XII, p. 429, 1909.

4. Philadelphia Medical Journal, Vol. XI, p. 137, 1903.

5. Journal of Pathology, Vol. XI, p. 135, 1906.

6. Guy's Hospital Report., Vol. LX, p. 211, 1906 


\section{The Fuocculant Precipitate Riaction}

A series of small test-tubes are prepared containing quantities of $\mathrm{N} / 1000$ sulphuric acid rising by $0 \cdot 1$ c.c. from 0.0 to $1 \cdot 2$ c.c., the total volume of liquid in each being made up to 2 c.c. with distilled water. Each tube then receives a drop (circa 0.02 c.c.) of blood; the contents are mixed and the tubes placed in a water bath at $45^{\circ} \mathrm{C}$. for one hour. They are then removed, wiped clean and dry on the outside and examined. The earlier tubes-i.e., those containing $0 \cdot 1$ and $0 \cdot 2$ c.c. $\mathrm{N} / 1000$ acid-appear little different from the control tube containing no acid; careful examination shows, however, that they are faintly opalescent. The liquid in the next few tubes is definitely opalescent, and the density of the opalescence increases progressively with increasing quantities of acid. With average normal human blood, one of the tubes containing between 0.7 and 0.9 c.c. N/1000 acid shows a further change in that the liquid is more or less occupied by a coarse flocculent precipitate. The coherence of the individual flocculi is so slight that by gently inverting the tube two or three times they are distributed into a uniform opalescence. In the great majority of cases the appearance of this precipitate occurs sharply and definitely, and affords an obvious objective end-point of the titration. It is very seldom that there is any doubt as to the tube in which the flocculent precipitate is added to the opalescence.

We are accustomed to take as the actual end-point the point half-way between the last tube which does not, and the first tube which does, show the precipitate. Increased accuracy is obtained by doing each experiment in triplicate: the precision of the reaction is such that the minute errors involved in measuring the acid and the blood sometimes result in some of the three tubes which are supposed to contain just the same mixture giving a positive result, while in the remainder no precipitate is produced.

It is convenient to distribute the blood along the series of tubes with a small dropping pipette. With an outside diameter at the point of about $1.2 \mathrm{~mm}$., a drop of normal human blood is rather more than 0.02 c.c. The exact volume is determined for each experiment by weighing five or ten drops and from the known specific gravity. Since the size of drop increases very rapidly as coagulation becomes imminent, it is desirable to take a series of drops for weighing both before and after distributing the blood in the test tubes, and to make the collection and 
distribution of the blood as expeditiously as may be. To ensure uniformity among the drops, the requisite amount of blood should be collected from the finger or ear in a small glass pot before it is taken into the pipette. There is a curious difference in the size of the drops which the same pipette will deliver from the blood of different individuals, all the samples showing the same content in haemoglobin. If the blood has been obtained quickly, and is altogether free from any suspicion of clotting, the variation in the size of each drop with different samples of normal blood containing approximately the same percentage of haemoglobin is not more than about 0.002 c.c., so that for many purposes, using a well-standardised pipette, weighing may be dispensed with. If one also knows approximately the answer which is to be expected, the amount of blood required may be still further reduced, and six drops ( 0.15 c.c.) will suffice.

Knowing the volume of the drops, the amount of acid required may be conveniently expressed either in terms of a standard drop of 0.02 c.c. or as c.c. of N/10 acid per 100 c.c. of blood. Thus with drops of normal blood weighing 0.0243 gms. $=0.023$ c.c., none of three tubes containing 0.7 c.c. $\mathrm{N} / 1000 \mathrm{H}_{2} \mathrm{SO}_{4}$ showed a precipitate which was present in two of three tubes containing 0.8 c.c. and in all three tubes containing 0.9 c.c. acid. The average amount of acid required was therefore 0.783 c.c. This is equivalent to 0.681 c.c. $\mathrm{N} / 1000$ acid for 0.02 c.c. blood or 34 c.c. N/10 acid per 100 c.c. blood. ${ }^{1}$ An error of 0.1 c.c. in the amount of acid required or of 0.003 c.c. in the volume of the drop of blood makes a difference of 5 c.c. N/10 acid per 100 c.c. of blood, or about 170 c.c. for the whole blood.

The end-point is ultimately the same at whatever temperature between $15^{\circ}$ and $45^{\circ}$ the reaction takes place. At the lower temperature, however, the flocculent precipitate takes much longer to appear and the end-point is not so sharply marked. At $34^{\circ}$ for example it takes two hours, at $20^{\circ}$ three hours to reach the same point as appears at $45^{\circ}$ in half an hour. The flocculent precipitate is well marked at $45^{\circ}$ in about thirty minutes, often in much less, and no alteration of the point at which it occurs takes place during the next four or five hours. The period of one hour at $45^{\circ}$, which we take as the standard time, is therefore sufficiently remote from the stage at which any rapid change occurs.

1. In the terminology which has often been used for the alkalinity of blood this is equal to 136 mgms. $\mathrm{NaOH}$ per 100 c.c. blood. 
The concentration of the acid, or the total volume to which the mixtures are made up, is, within wide limits, immaterial. Thus:-

\begin{tabular}{cccc}
$\begin{array}{c}\text { Total volume } \\
\text { of mixture }\end{array}$ & & \multicolumn{2}{c}{$\begin{array}{c}\text { c.c. of } \mathrm{N} / 1000 \text { acid required } \\
\text { Blood } \mathrm{A}\end{array}$} \\
$\mathbf{1}$ c.c. & $\ldots$ & 0.350 & 0.650 \\
2 c.c. & $\ldots$ & 0.367 & 0.650 \\
3 c.c. & $\ldots$ & 0.383 & 0.650 \\
4 c.c. & $\ldots$ & 0.350 & 0.650
\end{tabular}

Comparison of the different tubes is, however, facilitated if they are all about the same concentration, and we have found a total volume of 2 c.c. a convenient standard.

The nature of the flocculent precipitate. The reaction is given equally well by fresh, defibrinated, oxalated or citrated blood and by red corpuscles washed many times with salt solution. It is not given by citrated or oxalated plasma, by serum or by a solution of fibrin. If well-washed red cells are laked by adding distilled water equal in volume to the original plasma, and drops taken forthwith, a good reaction is obtained. But if the laked red cells are thoroughly centrifugalised, the supernatant fluid gives no reaction. If, on the other hand, the washed corpuscles are laked with $\mathrm{N} / 100 \mathrm{Na}_{2} \mathrm{CO}_{3}$, the supernatant fluid after centrifugalisation gives a fair flocculation, though not such a good reaction as before centrifugalisation. The reaction therefore depends on the presence of some part of the formed elements of the blood which does not go into solution on laking with distilled water. That the leucocytes are not concerned is probably shown by the fact that an emulsion of leucocytes free from red cells collected out of citrated blood gives no reaction. To be sure, however, that the reaction is not due to something in the leucocytes acting in conjunction with something in the red cells, it would be necessary to show that it is given by red cells free from leucocytes: this cannot be done, though the reaction seems to be just the same after as many leucocytes as possible have been removed. The precipitate is soluble in alkali or in excess of acid, about 1.4 c.c. N/500 acid being required for a drop of 0.02 c.c. The presumption, therefore, is that the precipitate is actually composed of the nucleoproteid of red cells. It seems, however, at least likely that the characteristic flocculation is in part connected with the presence of particles (stromata) on to which the precipitation occurs. 
Test experiments on blood-human or rabbit defibrinated ${ }^{1}$ - to which known acid or alkali had been added-gave fairly satisfactory results :c.c. $\mathrm{N} / 1000 \mathrm{H}_{2} \mathrm{SO}_{4}$ required for 0.02 c.c. blood

Lactic acid added

\begin{tabular}{|c|c|c|c|c|c|c|c|c|}
\hline & & \\
\hline $\begin{array}{c}\text { Calculated } \\
-0.149 \\
0.011 \\
0.270 \\
0.572 \\
0.713\end{array}$ & $\begin{array}{l}\text { Found } \\
0^{2} \\
0^{2} \\
0 \cdot 250 \\
0 \cdot 617 \\
0.717\end{array}$ & $\begin{array}{l}\text { Diff. } \% \\
=- \\
-\overline{8 \cdot 0} \\
+7 \cdot 3 \\
+0 \cdot 6\end{array}$ & $\begin{array}{c}\text { Calculated } \\
0.556 \\
0.709 \\
1.045 \\
1 \cdot 235\end{array}$ & $\begin{array}{l}\text { Found } \\
0 \cdot 583 \\
0 \cdot 717 \\
0.999 \\
1 \cdot 249\end{array}$ & $\begin{array}{l}\text { Diff. } \% \\
+4 \cdot 9 \\
+1 \cdot 1 \\
-4 \cdot 6 \\
+1 \cdot 1\end{array}$ & $\begin{array}{c}\text { Calculated } \\
0 \cdot 447 \\
0 \cdot 554 \\
0 \cdot 617 \\
0 \cdot 650 \\
0 \cdot 677 \\
0.974 \\
1 \cdot 194\end{array}$ & $\begin{array}{l}\text { Found } \\
0.450 \\
0.583 \\
0.583 \\
0.706 \\
0.683 \\
0.923 \\
1 \cdot 107\end{array}$ & $\begin{array}{l}\text { Diff. \% } \\
+0 \cdot 6 \\
+5 \cdot 2 \\
-5 \cdot 5 \\
+8 \cdot 6 \\
+0.9 \\
-5 \cdot 5 \\
-7 \cdot 8\end{array}$ \\
\hline
\end{tabular}

It is clear that the method may be extended to the examination of such fluids as blood serum by using blood or red cells as an indicator. In this way the distribution of the apparent alkalinity of whole blood between corpuscles and serum may be ascertained. It is then found that the serum in normal human blood accounts for from 1/10 to $1 / 5$, generally about $1 / 7$ of the total alkalinity as measured by this method; in other words the serum has an alkalinity of about 5 c.c. $\mathrm{N} / 10$ acid per cent. In comparing one blood with another, therefore, an accurate knowledge of the relative amounts of corpuscles and plasma is essential. Red cells washed repeatedly in salt solution become less and less alkaline as the washing proceeds.

It has been already indicated that the flocculation end-point treats added $\mathrm{Na}_{2} \mathrm{CO}_{3}$ as if it were alkali. But if defibrinated blood (which gives the same answer as the fresh blood from which it is prepared) is shaken up with $\mathrm{CO}_{2}$, the acidity is much increased.

Defibrinated blood

$0 \cdot 750$
$0 \cdot 483$ c.c. of $\mathrm{N} / 1000$ acid required for 0.02 c.c.

$\begin{array}{cccc} & \begin{array}{c}\text { Saturated with } \\ 5 \cdot 6 \% \mathrm{CO}_{2}\end{array} & & \begin{array}{c}\text { Saturated with } \\ \text { pure } \mathrm{CO}_{2}\end{array} \\ \ldots & 0.650 & \ldots & 0.250 \\ \ldots & 0.317 & \ldots & 0.083\end{array}$

Defibrinated blood which has been thoroughly pumped out in a vacuum gives the same result as before. treatment. The difference between arterial and venous blood is shown in the following figures giving the results of simultaneous measurements on blood from the carotid and large veins of rabbits under ether anaesthesia :c.c. $\mathrm{N} / 1000$ acid for 0.02 c.c. blood.

$\begin{array}{ccc}\text { Arterial } & & \text { Venous } \\ \mathbf{0 . 6 0 0} & \ldots & 0.557 \\ \mathbf{0 . 5 7 6} & \ldots & 0.494 \\ \mathbf{0 . 5 6 3} & \cdots & 0.467 \\ \mathbf{0 . 5 3 2} & \cdots & 0.488 \\ \mathbf{0 . 3 8 2} & \cdots & 0.304\end{array}$

1. Human gives a sharper end-point than rabbit blocd.

2. Flocculation occurred without addition of any acid. 
The average difference, therefore, is about 3 to 3.5 c.c. $\mathrm{N} / 10$ acid per 100 c.c. blood. A number of experiments showed that drops of blood obtained in the ordinary way gave the same results as samples taken from a moderately obstructed vein in the arm immediately afterwards.

Examples of the results obtained

(1) The time of day at which the blood is examined may have a considerable influence on the result. On first waking in the morning the blood is always more alkaline than during the active hours of laboratory work: e.g.

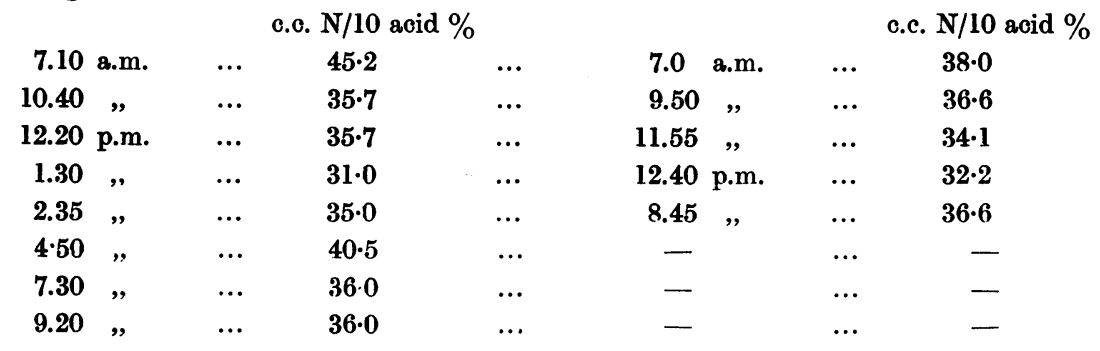

(2) Short sharp exercise (running, turning a pump handle) sufficient to produce marked dyspnoea increases the acidity:-

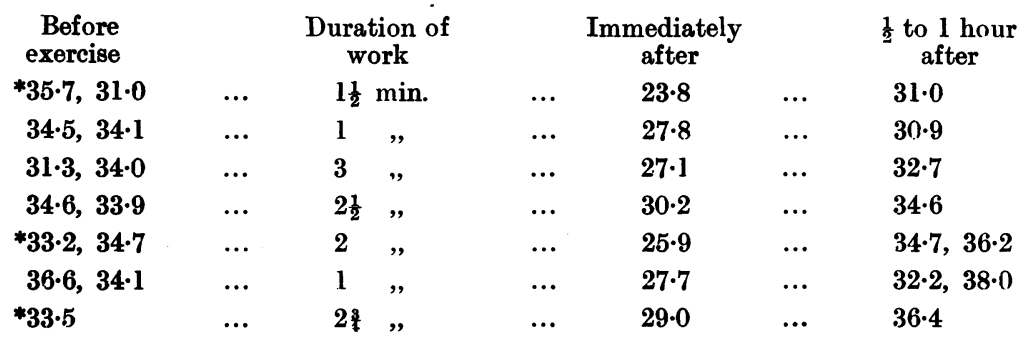

In the experiments marked * Dr. J. H. Ryffel was the subject. The exercise was in all cases sufficient to produce a definite increase in the lactic acid in the urine ${ }^{1}$. In the last experiment he also estimated the lactic acid in three 20 c.c. samples of blood taken from a vein in the arm, and found quantities equivalent to $1 \cdot 4,7 \cdot 9$ and $1 \cdot 8$ c.c. $N / 10$ acid per cent.; relatively these figures are in satisfactory agreement with our own obtained from the same samples.

Less violent muscular work, extending over a longer period, such as two hours' singles at tennis or walking eighteen miles in five hours, seems to have no effect which can be detected at the end of the exercise.

1. Proceedings Physiological Snciety. January, 1910. 
(3) We have not been able to find that meals have any influence. In one experiment, for instance, in which Dr. E. L. Kennaway was good enough to help us, the following results were obtained, a large mixed meal being eaten from 1 to 2 p.m. with good appetite:-

E. L. $\mathbf{K}$.

\begin{tabular}{|c|c|c|c|c|}
\hline \multicolumn{2}{|c|}{ Time } & \multicolumn{3}{|c|}{ c.c. $N / 10$ acid $\%$} \\
\hline 11.5 & a.m. & $\ldots$ & $32 \cdot 4$ & $\ldots$ \\
\hline 12.10 & p.m. & $\ldots$ & 33.9 & $\ldots$ \\
\hline 12.40 & , & $\ldots$ & $30 \cdot 2$ & $\ldots$ \\
\hline 2.0 & , & $\ldots$ & $29 \cdot 6$ & $\ldots$ \\
\hline 2.40 & , & $\ldots$ & $31 \cdot 6$ & $\ldots$ \\
\hline 3.15 & , & $\ldots$ & $27 \cdot 4$ & $\ldots$ \\
\hline 4.10 & " & $\ldots$ & $28 \cdot 9$ & $\ldots$ \\
\hline
\end{tabular}

A. E. B.

\begin{tabular}{cccc} 
Time & \multicolumn{3}{c}{ c.e. $\mathrm{N} / 10$ acid $\%$} \\
7.15 a.m. & $\ldots$ & $39 \cdot 4$ \\
$10.10 \quad$, & $\ldots$ & $35 \cdot 9$ \\
12.15 p.m. & $\ldots$ & $34 \cdot 4$ \\
2.5 & $\ldots$ & $\ldots$ & $31 \cdot 7$ \\
2.45 & $\cdots$ & $\ldots$ & 33.9 \\
3.25 & $\ldots$ & $\ldots$ & $34 \cdot 5$ \\
$4.5 \quad \#$ & $\ldots$ & $32 \cdot 4$
\end{tabular}

(4) A rabbit required 0.95 c.c. $\mathrm{N} / 1000$ acid for 0.023 c.c. blood; it was then poisoned with 90 c.c. 2 per cent. phosphoric acid per os. Three hours afterwards only 0.55 c.c. were required, and an hour later, when it was moribund, only 0.45 c.c.

\section{Colour Changes in Haemoglobin}

A. It was our original intention to use for the end-point the first visible change (from red to yellow) which is produced by acid in the colour of the haemoglobin. To investigate this, the mixtures of blood and acid, after one hour at $45^{\circ} \mathrm{C}$, are saturated with coal gas and centrifugalised until all opalescence and precipitate has been got rid of. Time and temperature are more practically important factors in this reaction than in the production of the flocculation: experiment showed, however, that no rapid change occurred in the neighbourhood of one hour if the reaction was going on at $45^{\circ} \mathrm{C}$. To ascertain the end-point exactly it is necessary to examine the tubes in a good open light, and it is very desirable to have the density of tint the same throughout by using tubes of equal diameter and making the total volumes of liquid exactly the same. Under these circumstances it was found in a number of experiments that the change of colour took place at the same point in the series as the flocculation. The former end-point is rather difficult and troublesome to make out: it was accordingly abandoned in farour of the more obvious flocculation. It is sensitive to added free $\mathrm{CO}_{2}$ in the same way.

B. For some reason or other it is easier to see the point at which successive additions of acid cease to make the haemoglobin any more yellow. With 0.02 c.c. of normal human blood, this point is reached 
with about 1.8 to 2.0 c.c. $\mathrm{N} / 500$ acid. To obtain by this means an expression of the alkalinity of the blood, the amount of acid consumed in converting the haemoglobin must be deducted, and it was therefore necessary to ascertain how much acid was required to convert a definite amount of haemoglobin into the yellow stuff (which may or may not be acid haematin). Haemoglobin crystals were prepared by various simple combinations of laking and cooling from horse corpuscles. The oxygen capacity of the solutions prepared from the fresh moist crystals was determined colorimetrically with a standard Gowers-Haldane haemoglobinometer and the absolute amount of haemoglobin calculated on the assumption-probably invalid-that 1 gramme of haemoglobin combines with 1.34 c.c. oxygen. On the same assumption it was found that haemoglobin constituted about 90 per cent. of the total solid matter in the solutions. These solutions were examined in series of tubes containing increasing quantities of acid as if they had been blood, being kept at $45^{\circ} \mathrm{C}$. for one hour. In five experiments we obtained the following results, each figure being the average of a number of determinations with different amounts of haemoglobin from each lot of crystals. The figures represent the number of c.c. of normal $\mathrm{H}_{2} \mathrm{SO}_{4}$ required to completely convert 1 gramme of haemoglobin into a yellow pigment in one hour at $45^{\circ} \mathrm{C}$.

$\begin{array}{r}1.13 \\ 1.01 \\ 1.09 \\ 1.06 \\ 1.08 \\ \hline \text { Average }=1.07 \text { c.c.1 }\end{array}$

From this figure the amount of acid consumed in converting the haemoglobin in any drop of blood may readily be computed. If, for example, the reading on the Gowers-Haldane haemoglobinometer is 72 per cent., then 0.02 c.c. of blood requires:-

$$
\frac{72 \times 18.5 \times 0.02 \times 1.07 \times 1000}{100 \times 1.34 \times 100}=2.13 \text { c.c. } N / 1000 \text { acid }
$$

In other words, for every 10 per cent. of haemoglobin on the scale of the haemoglobinometer, the haemoglobin in 0.02 c.c. of blood requires $0 \cdot 295$ c.c. $\mathrm{N} / 1000$ acid.

1. This suggests an equivalent of about 950 for haemoglobin if it is a mono acid base. 